\title{
Polarisation Effects in Optical Microresonators
}

\author{
M. Narizee Mohd Nasir*, Shahab Bakhtiari Gorajoobi, G. Senthil Murugan and Michalis N. Zervas \\ Optoelectronics Research Centre, University of Southampton, Southampton, SO17 1BJ, UK \\ mnmn1r11@soton.ac.uk
}

\begin{abstract}
Polarization effects in different types of optical microresonators are studied in detail. It is observed that microresonators with tighter axial power confinement, such as MBRs and micro-milled resonators, show larger cross-polarization coupling efficiencies.
\end{abstract}

\section{Introduction}

Over the years, a number of advanced high- $Q$ optical whispering gallery mode (WGM) microresonator-based devices have been proposed and successfully demonstrated [1]. Among the well-established WGM microresonators are the microsphere [2], microtoroid [3] and microdiscus [4]. Bottle microresonators (BMRs) [5] and bubble microresonators [6] have also been demonstrated recently with complex WGMs spectra. Since excitation of WGMs on any microresonator are polarization sensitive, it is important to understand their performance under TE/TM launching conditions and their polarization cross-coupling. It has been reported that light can change its state of polarization when it is coupled into and out of a microcavity [7]. A polarimetric setup was used in order to control the polarization and characterize the polarization cross-coupling in microspheres [8]. In this paper, we investigate the polarization cross-coupling behavior in a number of different microresonators, including a simple cylindrical microresonator [9], a new micro-rod resonator milled with a pulsed $\mathrm{CO}_{2}$ laser [10] and a BMR with cleaned-up spectrum (using FIB microgroove scars) [11].

\section{Polarization Cross-Coupling Charactrerization Set-Up}

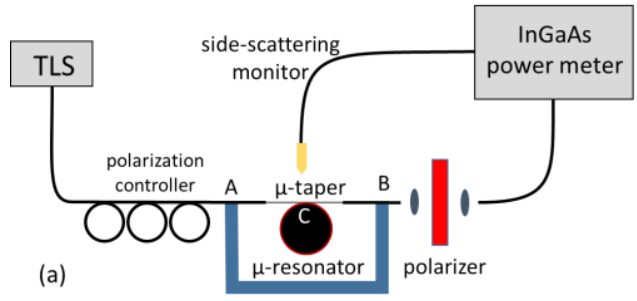

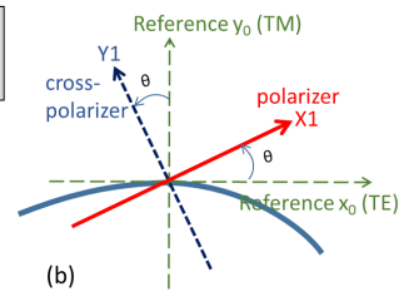

(b) schematic of reference axes and Fig. 1: (a) Polarization-resolved
polarizer/cross polarizer orientations

microresonator under test. A fiber polarization controller is used to adjust the polarization launched into the microresonators. The output end of the microtaper is cleaved close to its waist and kept straight in order to minimize any additional bending- and stress-induced birefringence. A polarizer is utilized at the output in order to facilitate polarization alignment and measure the induced polarization cross-coupling. In addition, a multimode pick-up fiber, in close proximity with the microresonator under test, is used to monitor scattered light and identify WGM resonances.
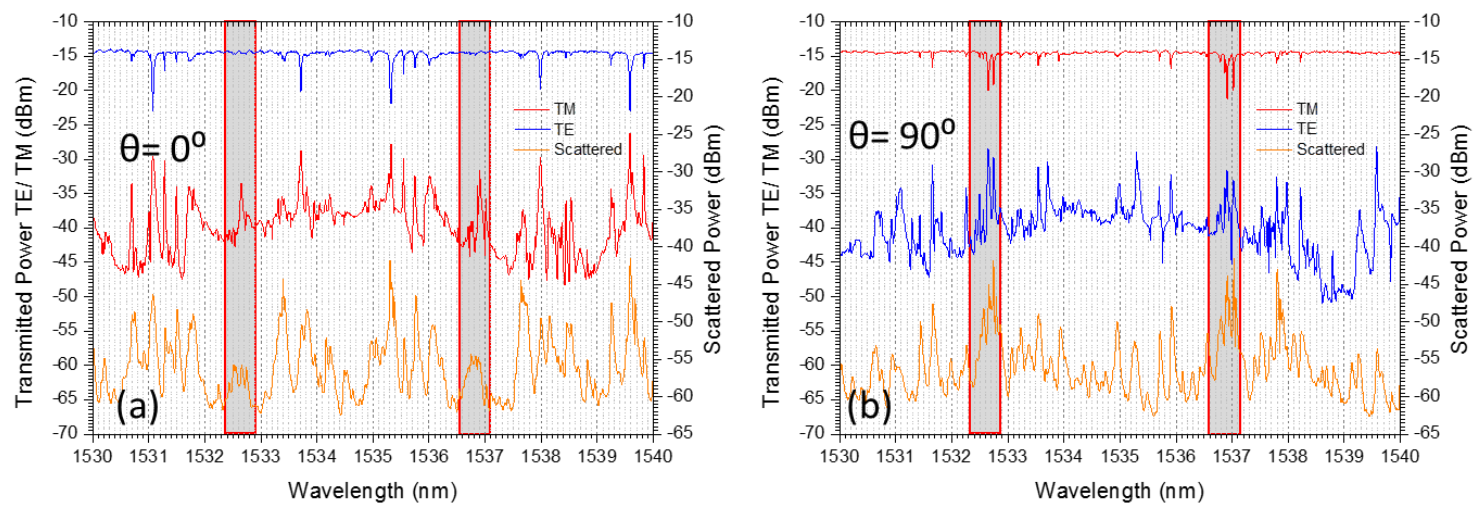

Fig. 2: Transmission spectra through the polarizer (top), cross-polarizer (middle) and scattered (bottom) for (a) $\mathrm{TE}\left(\theta=0^{\circ}\right)$, and (b) TM $\left(\theta=90^{\circ}\right)$ launching, for the micro-milled resonator. 
Fig. 2(a) and (b) show representative transmission spectra through the polarizer (top), cross-polarizer (middle) and scattered (bottom) for TE $\left(\theta=0^{\circ}\right)$ and $\operatorname{TM}\left(\theta=90^{\circ}\right)$ launching, respectively, for the micro-milled resonator [10]. The highlighted regions mark the positions of the TM resonances. It is observed that the cross-polarized (middle) and scattered (bottom) spectra show signatures of both TE and TM WGM resonances. The other two tested microresonators show also similar features. The spectra and the relative size of the cross-coupled and scattered resonance peaks were monitored with the change of the polarization offset angle $\theta$.
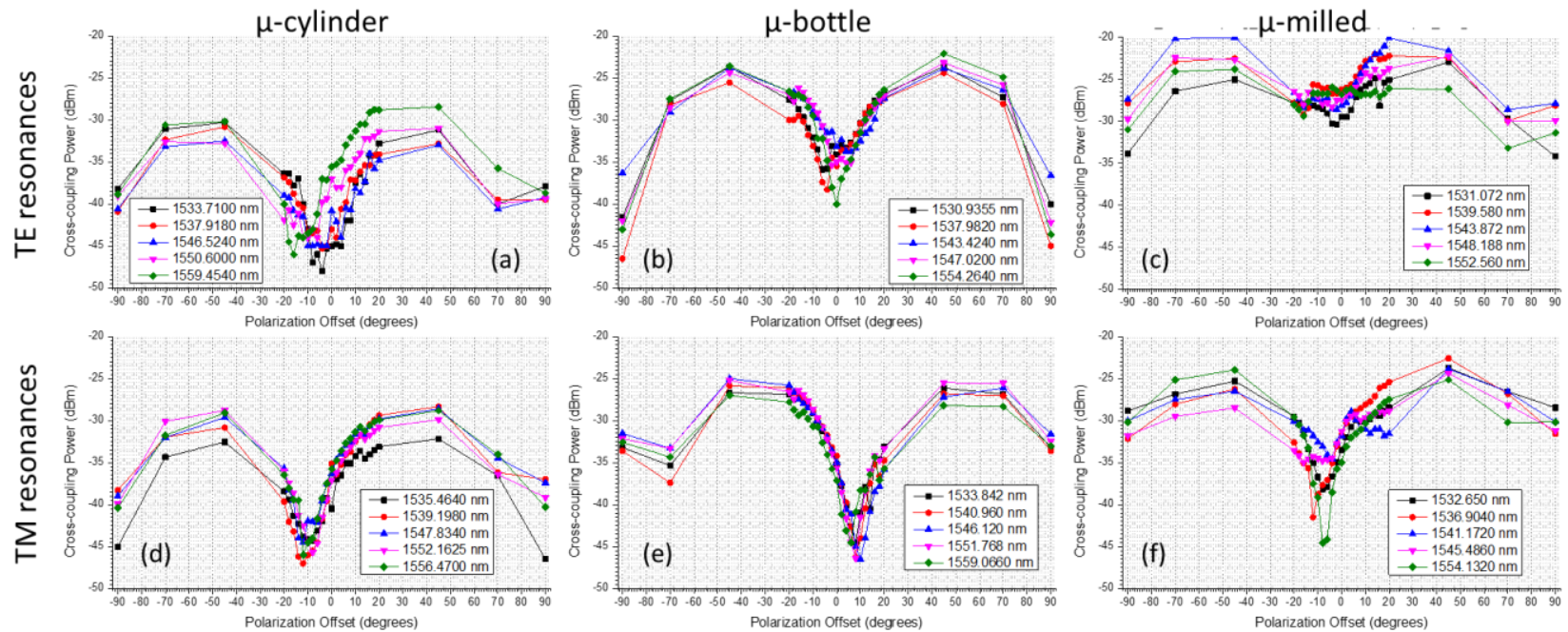

Fig. 3: Cross-polarization coupling results for (a)-(c) TE and (d)-(f) TM WGM resonances for micro-cylinder (a,d), microbottle (b,e) and micro-milled (c,f) resonators.

Figure 3 summarizes the results for the three tested microresonators. Figures 3(a)-(c) show the cross-coupled power variation with the polarization offset angle $\theta$ for the TE WGM resonances. Figures 3(d)-(f) correspond to the TM WGM resonances for micro-cylinder $(\mathrm{a}, \mathrm{d})$, microbottle $(\mathrm{b}, \mathrm{e})$ and micro-milled $(\mathrm{c}, \mathrm{f})$ resonator. All cross-polarization maxima were observed at about $\theta= \pm 45^{\circ}$. In MBRs and micro-milled resonators TE WGM resonances show in general larger cross-polarization efficiencies than the TM counterparts. In the case of the micro-cylinder resonator, on the other hand, the TE and TM resonance cross-polarization efficiencies are almost equal.

\section{Conclusions}

Cross-polarization coupling and scattered light spectra were obtained for three different optical microresonators for different polarization-offset launching angles. It is observed that microresonators with tighter axial power confinement, such as MBRs and micro-milled resonators, show larger cross-polarization coupling efficiencies and they should be preferred as polarization converters. In the case of micro-cylinder resonators, the lack of axial mode confinement results in the smallest and almost polarization independent cross-polarization coupling. Finally, it is shown that utilizing the TE WGM resonances results in superior cross-polarization performance.

\section{Acknowledgements:}

The work was supported by the EPSRC Centre for Innovative Manufacturing in Photonics.

\section{References}

[1] K. J. Vahala, Nature 424, 839-846 (2003).

[2] V. Lefevre-Seguin, Opt. Mater. 11, 153-165 (1999).

[3] D. K. Armani, T. J. Kippenberg, S. M. Spillane, and K. J. Vahala, Nature 421, 925-928 (2003)

[4] G. S. Murugan, J. S. Wilkinson, and M. N. Zervas, Appl. Phys. Lett. 101, 071106 (2012).

[5] G. S. Murugan, J. S. Wilkinson, and M. N. Zervas, Opt. Express 17, 11916-11925 (2009).

[6] M. Sumetsky, Y. Dulashako, and R. S. Windeler, Opt. Lett. 35, 898-900 (2010).

[7] G. Guan, and F. Vollmer, Appl. Phys. Lett. 86, 121115 (2005).

[8] P. Bianucci, C. R. Fietz, J. W. Robertson, G. Shvets, and C.-K. Shih, Opt. Express 15, 7000-7005 (2007).

[9] D. R. Rowland, and J. D. Love, IEEE Procedings, J. Optoelectronics 140, 177-188 (1993).

[10] S. B. Gorajoobi, G. S. Murugan, and M. N. Zervas, Conference on Lasers and Electro-Optics CLEO (2015), paper SF1H.7.

[11] M. Ding, G. S. Murugan, G. Brambilla and M. N. Zervas, Appl. Phys. Lett. 100, 081108 (2012). 